\title{
CIC-DUX4 oncoprotein drives sarcoma metastasis and tumorigenesis via distinct regulatory programs
}

\author{
Ross A. Okimoto, ${ }^{1,2}$ Wei Wu, ${ }^{1}$ Shigeki Nanjo, ${ }^{1}$ Victor Olivas, ${ }^{1}$ Yone K. Lin, ${ }^{1}$ Rovingaile Kriska Ponce, ${ }^{1}$ Rieko Oyama, ${ }^{3}$ \\ Tadashi Kondo, ${ }^{3}$ and Trever G. Bivona ${ }^{1,2,4}$ \\ 'Department of Medicine, ${ }^{2}$ Helen Diller Family Comprehensive Cancer Center, UCSF, San Francisco, California, USA. ${ }^{3}$ Division of Rare Cancer Research, National Cancer Center Research Institute, Tokyo, Japan. \\ ${ }^{4}$ Cellular and Molecular Pharmacology, UCSF, San Francisco, California, USA.
}

\begin{abstract}
Transcription factor fusion genes create oncoproteins that drive oncogenesis and represent challenging therapeutic targets. Understanding the molecular targets by which such fusion oncoproteins promote malignancy offers an approach to develop rational treatment strategies to improve clinical outcomes. Capicua-double homeobox 4 (CIC-DUX4) is a transcription factor fusion oncoprotein that defines certain undifferentiated round cell sarcomas with high metastatic propensity and poor clinical outcomes. The molecular targets regulated by the CIC-DUX4 oncoprotein that promote this aggressive malignancy remain largely unknown. We demonstrated that increased expression of ETS variant 4 (ETV4) and cyclin E1 (CCNE1) occurs via neomorphic, direct effects of CIC-DUX4 and drives tumor metastasis and survival, respectively. We uncovered a molecular dependence on the CCNE-CDK2 cell cycle complex that renders CIC-DUX4-expressing tumors sensitive to inhibition of the CCNE-CDK2 complex, suggesting a therapeutic strategy for CIC-DUX4-expressing tumors. Our findings highlight a paradigm of functional diversification of transcriptional repertoires controlled by a genetically aberrant transcriptional regulator, with therapeutic implications.
\end{abstract}

\section{Introduction}

The biological regulation of transcription factors and repressor proteins is an essential mechanism for maintaining cellular homeostasis, and is often dysregulated in human cancer (1). Indeed, chromosomal rearrangements involving transcriptional regulatory genes that lead to transcriptional dysregulation are present in many cancers, including approximately $30 \%$ of all soft tissue sarcomas (2). The majority of these oncogenic fusions involve transcription factors or regulators that are not readily "druggable" in a direct pharmacologic manner and thus have proven difficult to therapeutically target in the clinic. A prime example is the CIC-DUX4 fusion oncoprotein, which fuses capicua $(C I C)$ to the double homeobox 4 gene, DUX4. CIC-DUX4-positive soft tissue tumors are an aggressive subset of undifferentiated round cell sarcomas that arise in children and young adults. Despite histological similarities to Ewing sarcoma, CIC-DUX4-positive sarcomas are clinically distinct and typically characterized by the rapid development of lethal metastatic disease and chemoresistance (3). By revealing downstream molecular targets that relay the functional output of CIC-DUX4, we can potentially identify and develop a more rational therapeutic strategy to improve patient outcomes.

CIC is a transcriptional repressor protein (4). The CIC-DUX4 fusion structurally retains greater than $90 \%$ of native CIC, yet it

Conflict of interest: TCB is an advisor to Revolution Medicines, Novartis, AstraZeneca, Takeda, SpringWorks, Jazz, and Array BioPharma and receives research funding from Revolution Medicines and Novartis.

Copyright: (c) 2019, American Society for Clinical Investigation.

Submitted: November 21, 2018; Accepted: May 24, 2019; Published: July 22, 2019.

Reference information: J Clin Invest. 2019;129(8):3401-3406.

https://doi.org/10.1172/JCl126366. functions as a transcriptional activator instead of a transcriptional repressor $(5,6)$. This property suggests that the C-terminal DUX4 binding partner may confer neomorphic transcriptional regulatory properties to CIC, while retaining WT CIC DNA binding specificity. ETV4 is one of the most well-characterized transcriptional targets of CIC-DUX4 $(5,7,8)$. More than $90 \%$ of CIC-DUX4-expressing tumors show ETV4 upregulation by IHC, which distinguishes them from other small round blue cell sarcomas (8). We and others have demonstrated a prometastatic function for ETV4 overexpression in tumors with inactivation of WT CIC $(9,10)$. The functional role of ETV4 in CIC-DUX-positive tumors is unknown.

Beyond ETV4, the identity and function of other CIC target genes are less well defined. Intriguingly, recent studies showed increased expression of cell-cycle-regulatory genes in CIC-rearranged sarcomas, although the functional relevance of this observation for oncogenesis and cancer growth in this context is unclear $(6,7)$.

Here, we developed a range of in vitro and in vivo cancer model systems to define the mechanism by which CIC-DUX4 controls capicua-regulated transcriptional pathways to promote hallmark features of malignancy, including tumor cell survival, growth, and metastasis.

\section{Results and Discussion}

We recently reported that inactivation of native CIC derepresses an ETV4-MMP24-mediated prometastatic circuit (9). Since ETV4 is a direct transcriptional target of CIC-DUX4 (Supplemental Figure 1A; supplemental material available online with this article; https://doi.org/10.1172/JCI126366DS1) (6, 7), we hypothesized that the high metastatic rate observed in patients harboring CICDUX4 fusions was dependent on ETV4 expression. To explore this hypothesis, we developed an orthotopic soft tissue metastasis mod- 
A Orthotopic soft tissue sarcoma model
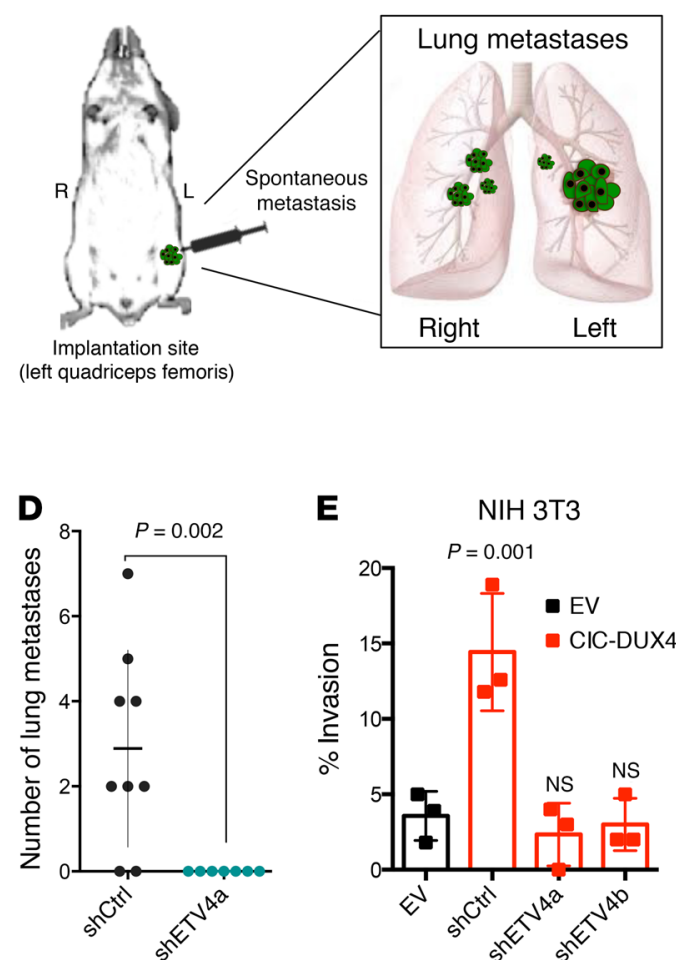

E

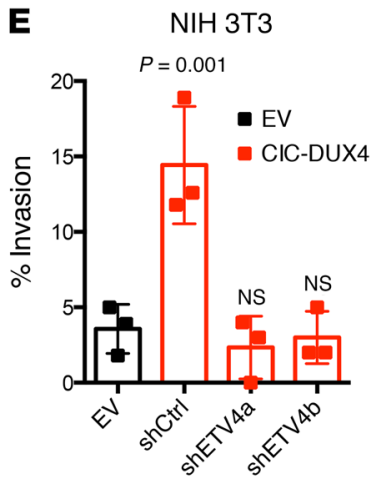

B

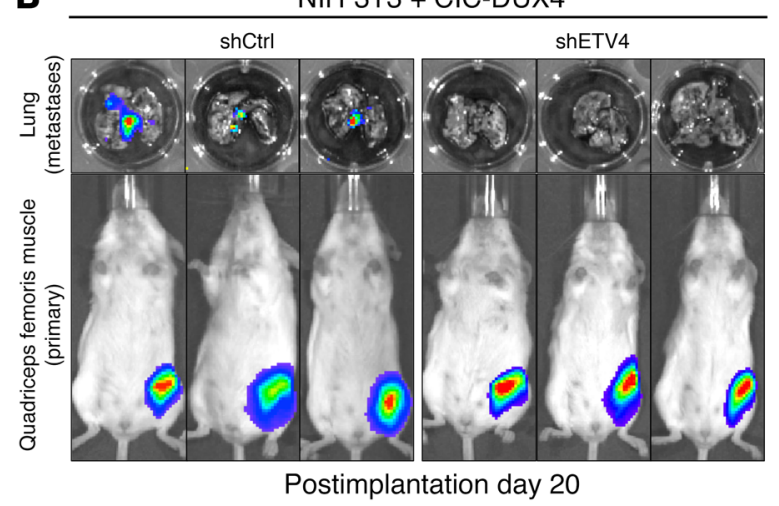

C

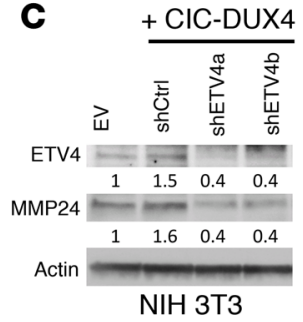

$\mathbf{F}$

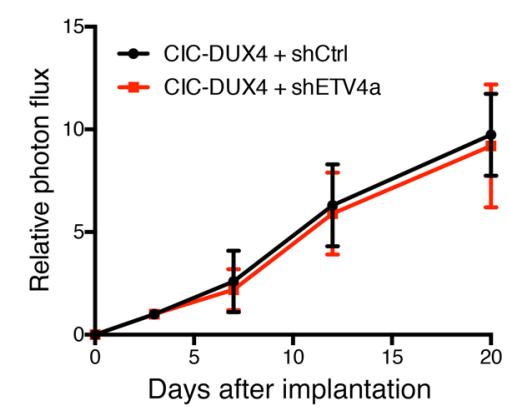

G

Subcutaneous xenograft

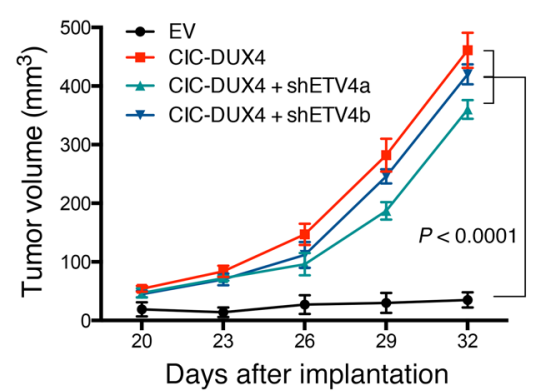

Figure 1. ETV4 promotes metastasis in CIC-DUX4-expressing sarcoma. (A) Schematic of the orthotopic soft tissue metastasis model. (B) Representative bioluminescence (BLI) images from mice bearing CIC-DUX4-expressing NIH 3T3 cells with shCtrl $(n=9)$ or shETV4 ( $n=7)$. (C) Immunoblot of ETV4 and MMP24 from NIH 3T3 cells expressing empty vector (EV), shCtrl, shETV4a, or shETV4b. (D) Number of lung metastases in mice bearing CIC-DUX4-expressing NIH 3T3 cells with shCtrl $(n=9)$ or shETV4 $(n=7)$. $P$ value, Student's $t$ test. (E) Transwell invasion assay comparing CIC-DUX4-expressing NIH 3T3 cells with EV, shCtrl, shETV4a, or shETV4b. $P$ value, 1-way ANOVA. Error bars represent SEM. (F) Relative photon flux from mice orthotopically implanted with CIC-DUX4-expressing NIH 3T3 cells expressing either shCtrl or shETV4. Error bars reflect SEM. (C) Subcutaneously implanted NIH 3T3 cells with EV, CICDUX4, or CIC-DUX4 plus shETV4a or shETVb. P value, 1-way ANOVA. Error bars represent SEM.

el utilizing luciferase-based imaging to track tumor dissemination in vivo (Figure 1A). This system produces rapid pulmonary metastases, accurately recapitulating metastatic tumor dissemination in patients (3). Using this in vivo system, we engineered NIH 3T3 mouse fibroblasts, which offer the advantage of a genetically controlled system (as with the study of other oncoproteins), to express the CIC-DUX4 fusion oncoprotein (11-13). We observed rapid primary tumor formation at the site of implantation in $100 \%$ of the injected mice (Figure 1B). Mice with genetic silencing of ETV4 showed decreased expression of its established target, MMP24 (9) (Figure 1C, and Supplemental Figure 1, B and C), and impaired metastatic efficiency in vivo and invasive capacity in vitro compared with mice bearing a control silencing vector (Figure 1, B-E). While ETV4 suppression decreased pulmonary metastases, it did not have a profound impact on tumor growth when CIC-DUX4expressing cells were implanted either orthotopically or subcutaneously into the flank of immunocompromised mice (Figure 1, F and G). These findings suggest that the primary function of CICDUX4-mediated ETV4 upregulation is to promote invasion and metastasis, but not tumor cell proliferation or tumor growth per se.

We next investigated transcriptional targets and programs that could regulate other key aspects of tumor biology beyond metastasis, including tumor cell proliferation and growth. Prior studies suggest- ed that the CIC-DUX4 fusion can regulate cell cycle gene expression $(6,7)$. We established that ectopic expression of CIC-DUX4 increased NIH 3T3 cell growth in vitro and in vivo through enhanced cell cycle progression (Supplemental Figure 2, A-E). The CIC-DUX4 fusion increased the number of cells progressing through S-phase, as reflected by an increased $\mathrm{G}_{2} / \mathrm{M}$ fraction compared with control (Supplemental Figure 2F). In order to identify CIC-DUX4-regulated cell cycle genes, we leveraged a publicly available microarray-based data set (GSE60740) to perform a comparative transcriptional analysis between CIC-DUX4-replete (IB120 EV cell line) and 2 independent CIC-DUX4-knockdown (KD) patient-derived cell lines (IB120 shCICDUX4a and IB120 shCIC-DUX4b) (14). Notably, in the context of the CIC-DUX4 fusion, CIC retains it binding specificity but acquires activating properties (5). Hence, we focused our attention on genes that were downregulated upon CIC-DUX4 KD. We observed 165 shared downregulated (and 105 upregulated) genes between the 2 independent shCIC-DUX4 data sets (IB120 shCIC-DUX4a and IB12O shCICDUX4b) (Figure 2A, and Supplemental Tables 1 and 2). Functional clustering of the 165 putative CIC-DUX4 target genes revealed enrichment for genes that regulate DNA replication and cell cycle machinery (Figure 2B) (15). We compared the expression of these 165 genes in 14 CIC-DUX4 and 7 EWS-NFATC2 patient-derived tumors that were profiled on the same Affymetrix array (GSE60740). We observed a 
A

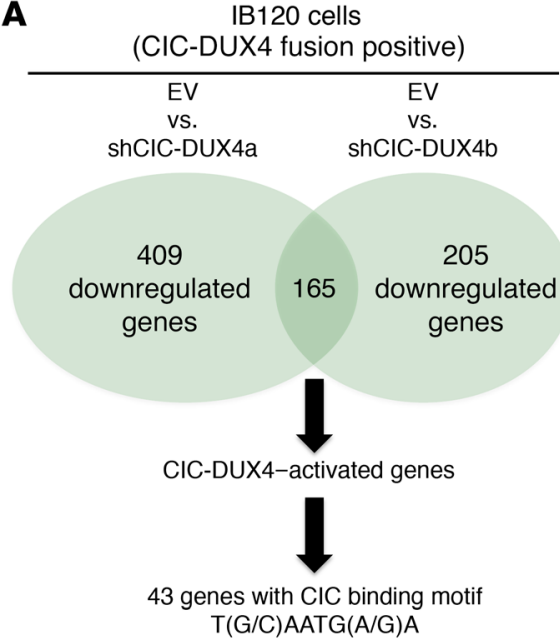

B

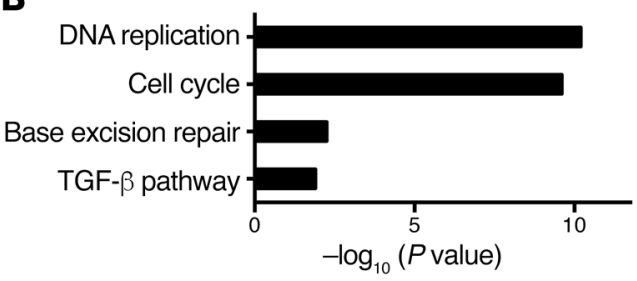

C
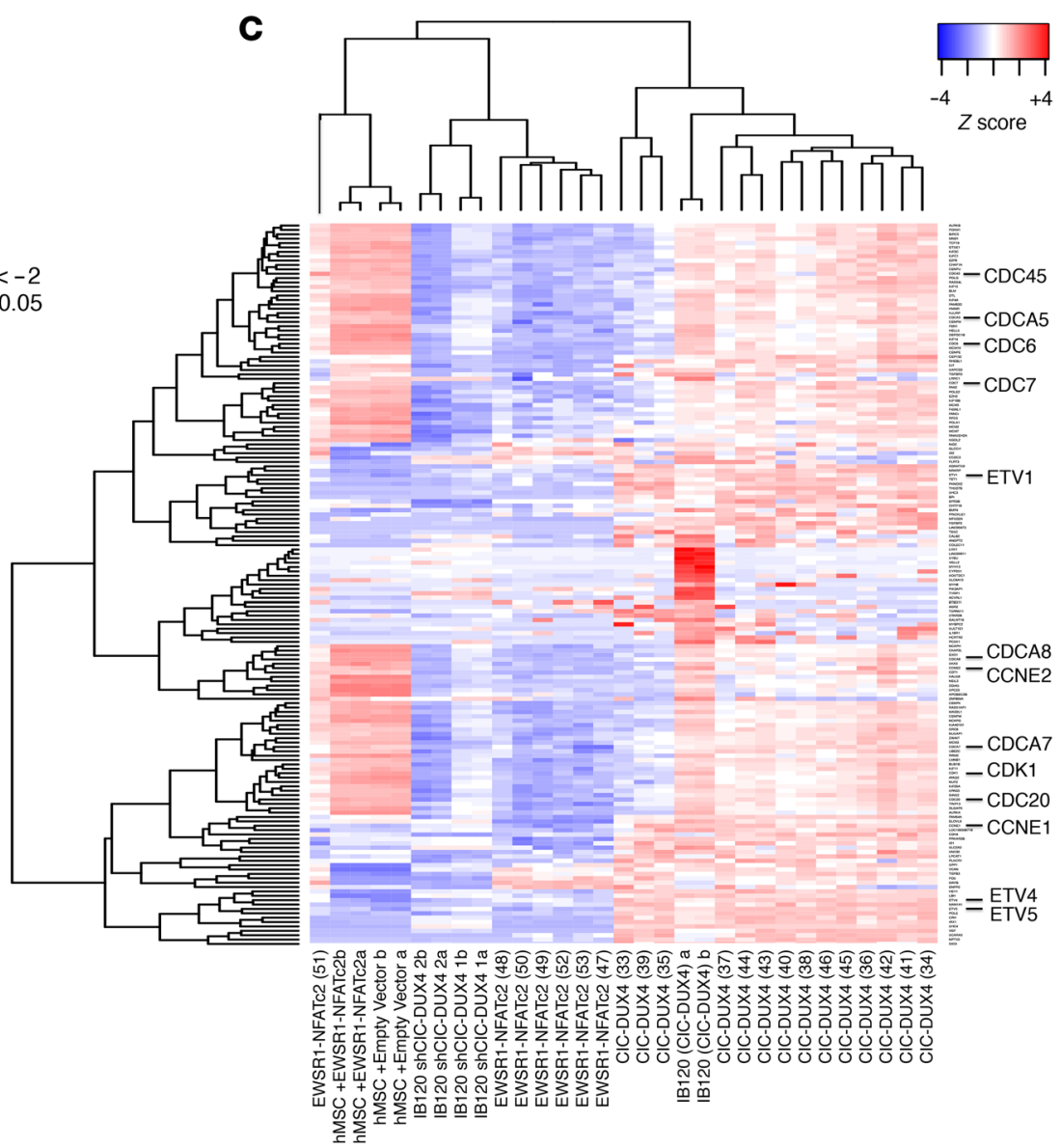

$\log \mathrm{FC}<-2$

FDR $<0.05$

Figure 2. CIC-DUX4 regulates cell cycle genes. (A) Schematic algorithm to identify putative CIC-DUX4 target genes. (B) Functional annotation of CICDUX4-activated genes in IB120 cells. (C) Heatmap depicting the 165 CIC-DUX4-activated genes identified in A across 14 CIC-DUX4 and 7 EWSR1-NFATc2 patient-derived tumors. Cell cycle gene symbols are magnified.

significant increase in the expression of multiple cell-cycle-regulatory genes in CIC-DUX4-expressing tumors (Figure 2C). Our findings extend recent studies $(6,7)$ and indicate that targeting the cell cycle in CIC-DUX4-expressing tumors is a potential therapeutic approach.

In order to identify direct transcriptional targets of the CICDUX4 fusion, we first surveyed the promoters (within $-2 \mathrm{~kb}$ and +150 bp of the transcription start site) of all 165 putative response genes for the highly conserved CIC-binding motif (T[G/C]AATG[A/G]A) (5). Using this systematic approach, we identified 43 genes, including the known CIC target genes ETV1, -4 , and -5 and multiple regulators of the cell cycle (Supplemental Table 3). Since ectopic expression of CIC-DUX4 promoted S-phase progression (Supplemental Figure 2, E and F), we focused on genes that directly regulate the $\mathrm{G}_{1} / \mathrm{S}$ transition. Of these genes, cyclin E1 (CCNE1) expression was consistently upregulated in CIC-DUX4-expressing tumors (Figure 2C). We therefore investigated whether CIC-DUX4 transcriptionally controls CCNE1 expression. To explore this hypothesis, we first localized 2 tandem CIC-binding motifs within $1 \mathrm{~kb}$ of the CCNE1 transcriptional start site (Figure 3A). We next performed ChIP-PCR analysis, which revealed CCNE1 promoter occupancy by the CICDUX4 fusion (Figure 3, B and C). Additionally, a luciferase-based reporter assay demonstrated enhanced promoter activity by the ectopic expression of CIC-DUX4 compared with CIC WT and emp- ty vector control (Figure 3D). These data show that $C C N E 1$ is a direct transcriptional target that is upregulated by CIC-DUX4.

To explore the functional role of CCNE1 in CIC-DUX4-expressing tumors, we genetically silenced CCNE1 in CIC-DUX4-expressing NIH 3T3 cells (a genetically controlled system) and observed decreased growth in vitro and in vivo compared with control (Figure 3, E-G, and Supplemental Figure 3, A-C). While there is no validated pharmacologic strategy to directly block CCNE1, inhibition of the CCNE1 binding partner CDK2 has therapeutic efficacy in other cancer types (16-18). We hypothesized that inhibiting CDK2 in CIC-DUX4-expressing cells with the established small molecule drug dinaciclib (16) could limit tumor growth. To explore this hypothesis, we implanted CIC-DUX4-expressing NIH 3T3 cells subcutaneously into immunodeficient mice and treated them with low-dose dinaciclib $(20 \mathrm{mg} / \mathrm{kg} / \mathrm{d})$; we observed decreased growth compared with vehicle control (Figure 4, A-C) (16). These findings suggest that pharmacologic inhibition of the CCNE-CDK2 complex is a potential therapeutic strategy in CIC-DUX4-expressing tumors.

There are few patient-derived models of CIC-DUX4-positive tumors. Nevertheless, in order to increase the clinical relevance of our findings we obtained rare, established patient-derived CICDUX4-expressing cells (NCC_CDS1_X1 and NCC_CDS1_X3) (19) to test the functional impact of ETV $4 \mathrm{KD}$ and $\mathrm{CDK} 2$ inhibition. 

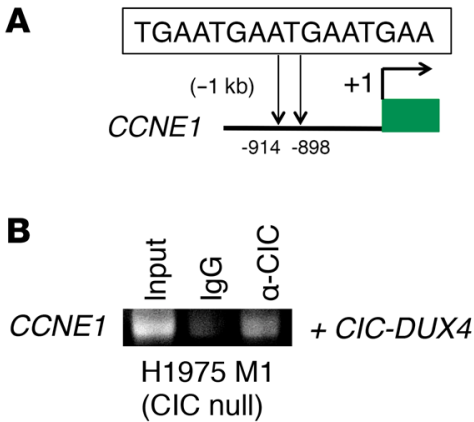

E

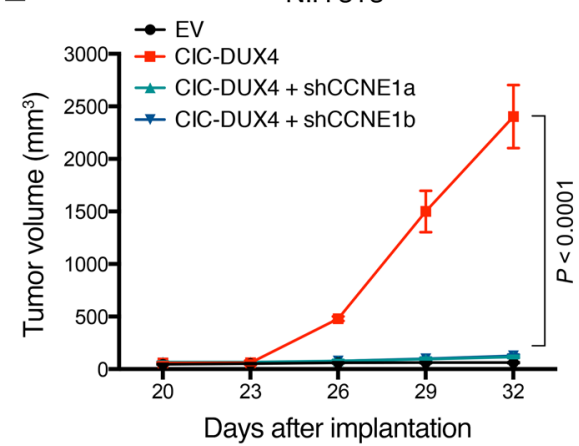

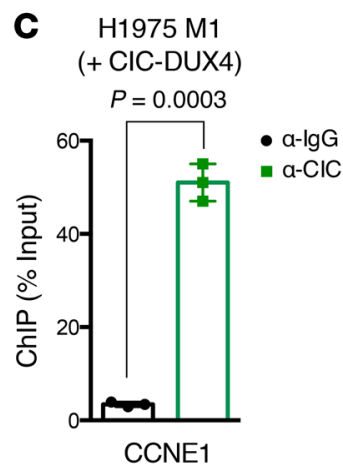

F $\mathrm{NIH}_{3} \mathrm{~T} 3+\mathrm{CIC}-\mathrm{DUX} 4$

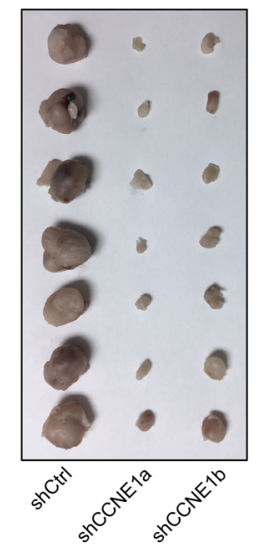

D
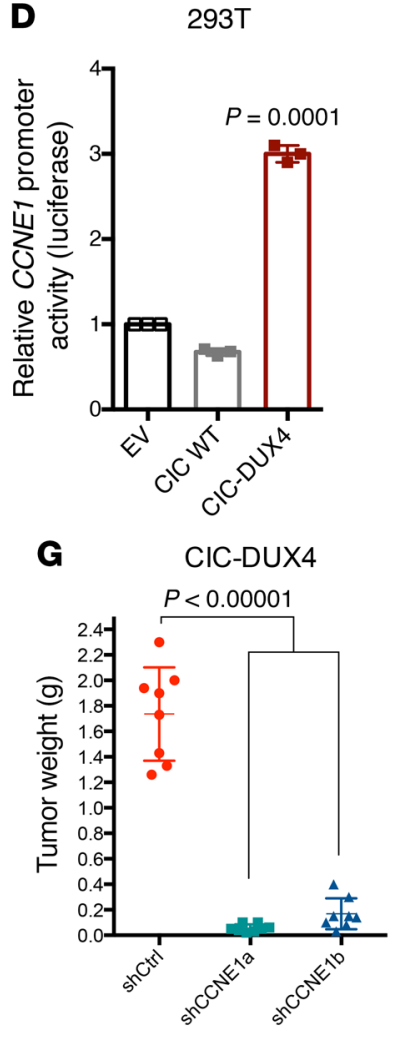

Figure 3. CCNE1 is a molecular target of CIC-DUX4. (A) Schematic of 2 CIC-binding motifs in the CCNE1 promoter. (B and C) ChIP-PCR from H1975 M1 (CIC null) cells reconstituted with CIC-DUX4, showing CIC-DUX4 occupancy on the CCNE1 promoter. $P$ value, Student's $t$ test. Error bars represent SEM. Performed in triplicate. (D) CCNE1 luciferase promoter assay in 293T cells comparing EV with CIC WT or CIC-DUX4. $P$ value, 1-way ANOVA. Error bars represent SEM. Performed in triplicate. (E) Subcutaneously implanted NIH 3T3 cells expressing EV, CIC-DUX4 $(n=7)$, or CIC-DUX4 plus shCCNE1a ( $n$ $=7)$ or shCCNE1b $(n=7)$. $P$ value, 1-way ANOVA. Error bars represent SEM. (F) Tumor explants from mice in E. (C) Tumor weights from mice in $\mathbf{E}$ $P$ values, 1-way ANOVA.

Ewing sarcoma), in vitro or in vivo (Supplemental Figure 6, A and B). Consistent with these findings, we observed low levels of CCNE1 in PAX3-FOXO1-positive RH30 rhabdomyosarcoma cells compared with CIC-DUX4-expressing NCC_ CDS1_X1 cells (Supplemental Figure 6C). These findings further sug-

We found that genetic silencing of ETV4, but not ETV1 or ETV5 (known CIC-DUX4 target genes) (5), decreased invasiveness but did not impact tumor growth or apoptosis (Supplemental Figure 4, A-G). Additionally, we found that CIC-DUX4-expressing cells were exquisitely sensitive to nanomolar (nM) concentrations of 2 established, independent CDK2 inhibitors, dinaciclib and SNS-032 $(16,20)$ (Figure 4D and Supplemental Figure 5A). The effects on tumor viability were largely mediated through apoptotic cell death as measured by poly(ADP-ribose) polymerase (PARP) cleavage and caspase activity, again indicating that CIC-DUX4expressing tumors are dependent on the CCNE-CDK2 complex for survival (Figure 4, E-G, and Supplemental Figure 5, B and C). To mitigate the off-target effects of dinaciclib and SNS-032, we performed genetic silencing of CDK7 and CDK9, which did not suppress NCC_CDS1_X1 growth (Supplemental Figure 5, D-G).

We next tested whether CDK2 inhibition could specifically suppress the growth of patient-derived CIC-DUX4-expressing cells in vivo. To address this hypothesis, we generated subcutaneous tumor xenografts of the patient-derived CIC-DUX4-expressing cells in immunodeficient mice and found that dinaciclib limited tumor growth compared with vehicle control treatment (Figure 4, H-J). The decreased tumor growth observed in dinaciclib-treated mice was accompanied by increased PARP cleavage in tumor explants, consistent with the apoptotic effect observed in vitro (Figure $4 \mathrm{~K}$ ). The impact on tumor growth following CDK2 inhibition was relatively specific for CIC-DUX4-expressing tumors, as we did not observe a significant response to dinaciclib in other sarcoma subtypes that harbor distinct transcription factor fusion oncoproteins (rhabdomyosarcoma or gest that CIC-DUX4-expressing tumors are molecularly dependent on the CCNE-CDK2 complex, which is a potential therapeutic target.

To further demonstrate the therapeutic specificity of targeting CDK2, we used the clinical CDK4/6 inhibitor palbociclib, which has no substantial activity against CDK2, and found no impact on tumor growth or apoptosis in NCC_CDS1_X1 cells (Supplemental Figure 7A). Moreover, while genetic silencing of CDK2 with siRNAs did not impact cell invasion, it did decrease cell number and viability in CICDUX4-expressing NCC_CDS1_X1 cells compared with control (Supplemental Figure 7, B-E). While we did not observe an effect on viability with CCNE1 KD alone, combined CCNE1 and CCNE2 KD reduced viability to similar levels as siCDK2 cells (Supplemental Figure 7, C, $\mathrm{D}, \mathrm{F}$, and $\mathrm{G}$ ). These findings are consistent with prior observations that both CCNE1 and CCNE2 converge on and activate CDK2, which our pharmacologic and genetic studies described above indicate is required for CIC-DUX4-expressing tumor survival (21).

Our data suggest that in human-derived NCC_CDS1_X1 cells, CCNE2 can compensate for CCNE1 loss. Consistent with this notion, we observed approximately 100 -fold upregulation of CCNE2 mRNA upon genetic silencing of CCNE1 and through ChIP-Seq analysis identified CIC-DUX4 binding to the promoter of CCNE1 but not CCNE2 (Supplemental Figure 8, A-C) in human NCC_CDS1_X1 cells. In contrast to these findings, we did not observe a compensatory increase in CCNE2 mRNA with CCNE1 KD in NIH 3T3 (mouse) cells expressing CIC-DUX4 (Supplemental Figure 8, D and E). The lack of CCNE2 upregulation in CIC-DUX4-expressing NIH 3T3 cells is a plausible explanation for the growth-suppressive effect of CCNE1 KD in our initial NIH 3T3 system (Figure 3E), a phenotype that was 
A

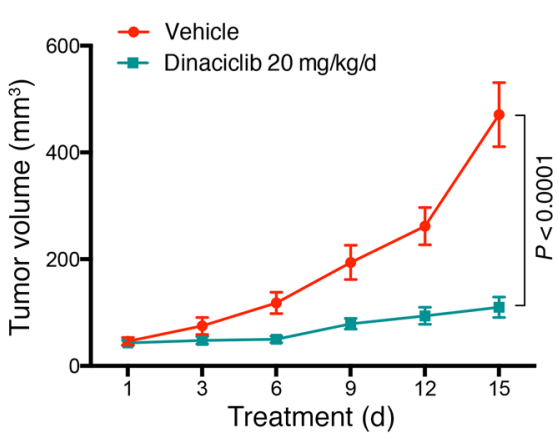

B

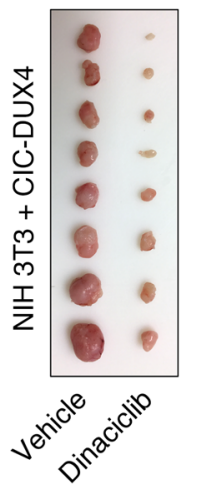

E

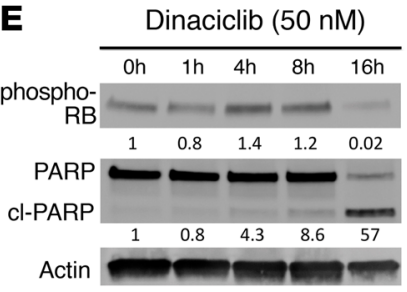

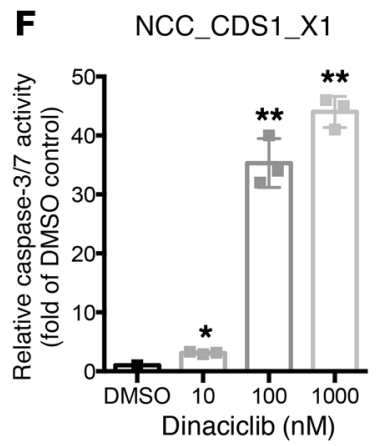

C
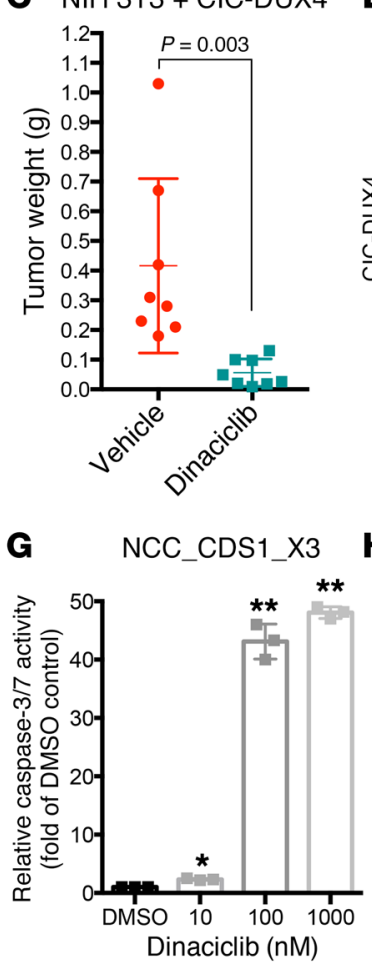

D

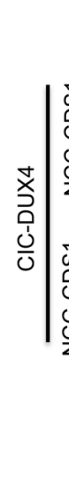

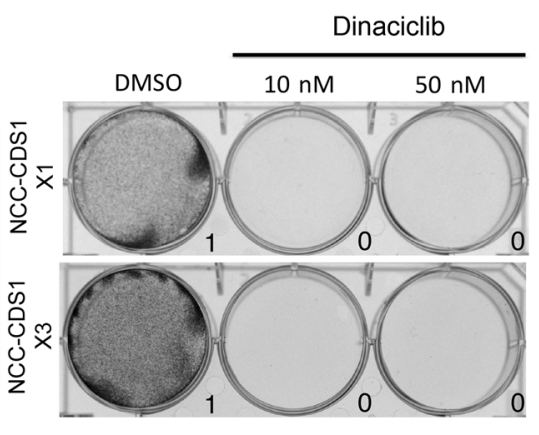
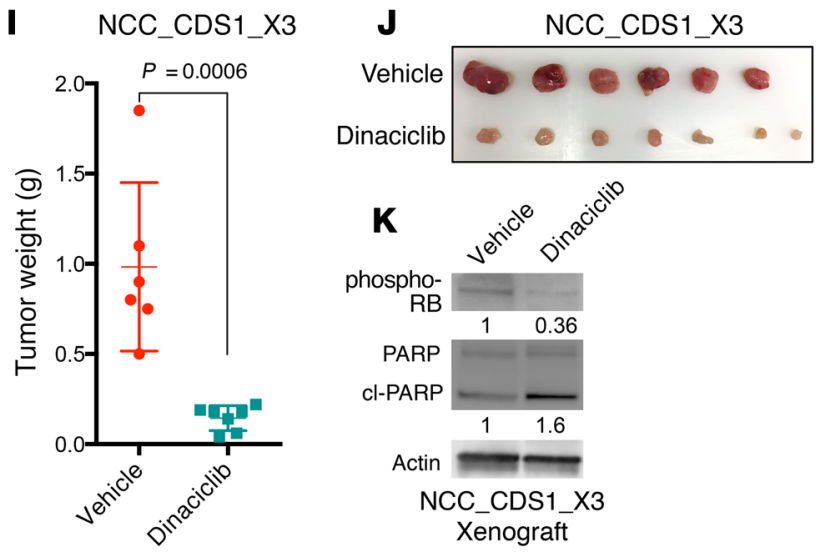

Figure 4. The CCNE-CDK2 complex is a therapeutic target in CIC-DUX4 sarcoma. (A) Subcutaneously implanted NIH 3T3 cells expressing CIC-DUX4 and treated with vehicle $(n=8)$ or dinaciclib $(n=8)$. $P$ value, Student's $t$ test. Error bars represent SEM. (B) Tumor explants from mice in A. (C) Tumor weights of mice in A. $P$ value, Student's $t$ test. (D) Patient-derived CIC-DUX4-expressing cells (NCC_CDS1_X1 and NCC_CDS1_X3) treated with dinaciclib or DMSO. Performed in duplicate. (E) Immunoblot of phosphorylated retinoblastoma (RB), PARP, and actin control in NCC_CDS1_ $\mathrm{X} 3$ cells treated with dinaciclib. Representative figure; performed in duplicate. (F) Relative caspase-3/7 activity in NCC_CDS1_X1 and (C) NCC_CDS1_X3 cells treated with dinaciclib or DMSO. ${ }^{*} P=0.01,{ }^{* *} P<0.0001,1$-way ANOVA. Error bars represent SEM. (H) Subcutaneously implanted NCC_CDS1_X3 cells treated with vehicle control $(n=6)$ or dinaciclib ( $n=7)$. $P$ value, Student's $t$ test. Error bars reflect SEM. (I) Tumor weights from mice treated in $\mathbf{H}$. $P$ value, Student's $t$ test. (J) Tumor explants from mice in $\mathbf{H}$. (K) Immunoblot of phosphorylated RB, total and cleaved PARP, and actin control from a NCC_CDS1_X3 tumor explant treated with vehicle (Veh) or dinaciclib. Representative figure; performed in duplicate.

not shared with human-derived NCC_CDS1 cells (Supplemental Figure 7, C and D). Importantly, we identified CIC-DUX4-binding sites in the promoter of mouse CCNE1, but not CCNE2 ( $-4 \mathrm{~kb}$ to +100 of the transcription start site), suggesting that CCNE1 (but not CCNE2) is a conserved transcriptional target in human (NCC_CDS1) and mouse (NIH 3T3 expressing CIC-DUX4) cells (Supplemental Tables 3 and 4 , and Supplemental Figure 8F). Our mechanistic dissection of CIC-DUX4-expressing tumors reveals a unique molecular and therapeutic dependence on the CCNE-CDK2 cell cycle complex. Our data show that this dependence can be exploited with clinical CDK2 inhibitors that limit tumor growth through apoptotic induction in a tumor type with few effective therapies.

The CIC-DUX4 fusion oncoprotein is a relatively understudied molecular entity that characterizes a rare but lethal subset of undifferentiated round cell sarcomas. We undertook a mechanistic dissection of the molecular function of the CIC-DUX4 fusion oncoprotein and report unique dependencies on specific transcriptional targets of the fusion oncoprotein that promote either tumor growth or metastasis. We reveal that ETV4 is a conserved target gene that enhances the metastatic capacity of CIC-DUX4-expressing tumors, without significantly impacting tumor growth. These findings extend previous data that demonstrate a prometastatic role for ETV4 in certain human cancers $(9,22)$. Targeting an ETV4-mediated transcriptional program downstream of CIC-DUX 4 can potentially limit tumor dissemination.

Further analysis of CIC-DUX4-bearing tumors coupled with our functional studies also identified a dependence on specific cell cycle machinery to enhance tumor growth but not invasive capacity. We reveal that the CCNE-CDK2 complex is a molecular target of the CIC-DUX4 oncoprotein that controls tumor growth and survival. While others have observed transcriptional upregulation of cell cycle genes in CIC-DUX4-expressing tumors $(6,7)$, our data establish the CCNE-CDK2 complex as a direct molecular target that can be pharmacologically exploited with clinically developed CDK2 inhibitors. The therapeutic impact may extend beyond the CIC-DUX4 fusion 
to include other CIC-fused oncoproteins. Recent findings reveal a shared transcriptional program downstream of all known CIC fusions, including CIC-FOXO4 and CIC-NUTM1 (23). These findings suggest that many CIC-fused tumors retain their CIC-binding specificity while converting native CIC into a transcriptional activator instead of a repressor in a neomorphic manner. It would be compelling to explore whether the CCNE-CDK2 complex or other components of the cell cycle machinery drive tumor growth in these other CIC-fused tumor types, an area for future investigation.

Our mechanistic dissection of the downstream molecular targets of CIC-DUX4 provides therapeutically relevant insight for targeting ETV4-mediated metastasis and CCNE-CDK2-regulated tumor growth. Our data support a broader conceptual paradigm in which certain transcription factor fusion oncoproteins, such as CIC-DUX4, utilize neomorphic and distinct downstream regulatory programs to control divergent cancer hallmarks, such as proliferative capacity and metastatic competency. The data offer a potential mechanistic explanation for the pleiotropic functions of this important class of oncoproteins (i.e., transcription factor fusion oncoproteins such as CIC-DUX4).

Our findings highlight the clinical importance of molecular subclassification of morphologically similar tumor types, such as small round cell sarcomas. Identifying the different fusion oncoproteins present in clinical samples paves the way for oncoprotein fusion-specific therapeutic targeting to improve patient outcomes. Our study highlights the utility of elucidating the mechanistic features of tumors that are driven by transcription factor fusion oncoproteins to identify precision medicine-based molecular therapeutic strategies.

\section{Methods}

Refer to Supplemental Methods for details.
Statistics. Experimental data are presented as mean \pm SEM. $P$ values derived for all in vitro experiments were calculated using 2-tailed Student's $t$ test or 1-way ANOVA. $P<0.05$ was considered statistically significant.

Study approval. For tumor xenograft studies, including orthotopic and subcutaneous models, specific pathogen-free conditions and facilities were approved by the American Association for Accreditation of Laboratory Animal Care. Surgical procedures were reviewed and approved by the UCSF IACUC, protocol AN107889-03A.

\section{Author contributions}

RAO designed and performed the experiments, analyzed the data, and wrote the manuscript. RAO, VO, SN, YKL, RKP, RO, and TK performed in vitro and in vivo mouse studies. WW performed bioinformatics. TK performed experiments and provided cell lines. TGB directed the project, analyzed experiments, and wrote the manuscript.

\section{Acknowledgments}

RAO was supported by a grant from the A.P. Giannini Foundation and NIH 1K08CA222625-01. TGB acknowledges support from NIH/NCI U01CA217882, NIH/NCI U54CA224081, NIH/NCI R01CA204302, NIH/NCI R01CA211052, NIH/NCI R01CA169338, and the Pew and Stewart Foundations.

Address correspondence to: Ross A. Okimoto, 513 Parnassus Avenue, HSW1201, San Francisco, California 94143, USA. Phone: 415.514.1083; Email: ross.okimoto@ucsf.edu. Or to: Trever G. Bivona, 600 16th Street, Genentech Hall, N232, San Francisco, California 94158, USA. Phone: 415.502.0237; Email: trever.bivona@ucsf.edu.
1. Bhagwat AS, Vakoc CR. Targeting transcription factors in cancer. Trends Cancer. 2015;1(1):53-65.

2. Mertens F, Antonescu CR, Mitelman F. Gene fusions in soft tissue tumors: recurrent and overlapping pathogenetic themes. Genes Chromosomes Cancer. 2016;55(4):291-310.

3. Antonescu CR, et al. Sarcomas with CIC-rearrangements are a distinct pathologic entity with aggressive outcome: a clinicopathologic and molecular study of 115 cases. Am J Surg Pathol. 2017;41(7):941-949.

4. Jiménez G, Shvartsman SY, Paroush Z. The Capicua repressor - a general sensor of RTK signaling in development and disease. J Cell Sci. 2012;125(pt 6):1383-1391.

5. Kawamura-Saito M, et al. Fusion between CIC and DUX4 up-regulates PEA3 family genes in Ewing-like sarcomas with $\mathrm{t}(4 ; 19)$ (q35;q13) translocation. Hum Mol Genet. 2006;15(13):2125-2137.

6. Specht K, Sung YS, Zhang L, Richter GH, Fletcher CD, Antonescu CR. Distinct transcriptional signature and immunoprofile of CIC-DUX4 fusion-positive round cell tumors compared to EWSR1-rearranged Ewing sarcomas: further evidence toward distinct pathologic entities. Genes Chromosomes Cancer. 2014;53(7):622-633.

7. Yoshimoto T, et al. CIC-DUX4 induces small round cell sarcomas distinct from ewing sarcoma. Cancer Res. 2017;77(11):2927-2937.

8. Le Guellec S, Velasco V, Pérot G, Watson S, Tirode F, Coindre JM. ETV4 is a useful marker for the diagnosis of CIC-rearranged undifferentiated round-cell sarcomas: a study of 127 cases including mimicking lesions. Mod Pathol. 2016;29(12):1523-1531.

9. Okimoto RA, et al. Inactivation of Capicua drives cancer metastasis. Nat Genet. 2017;49(1):87-96.

10. Kim E, et al. Capicua suppresses hepatocellular carcinoma progression by controlling the ETV4MMP1 axis. Hepatology. 2018;67(6):2287-2301.

11. May WA, et al. The Ewing's sarcoma EWS/ FLI-1 fusion gene encodes a more potent transcriptional activator and is a more powerful transforming gene than FLI-1. Mol Cell Biol. 1993;13(12):7393-7398.

12. Lam PY, Sublett JE, Hollenbach AD, Roussel MF. The oncogenic potential of the Pax3-FKHR fusion protein requires the Pax3 homeodomain recognition helix but not the Pax3 paired-box DNA binding domain. Mol Cell Biol. 1999;19(1):594-601.

13. Barco R, et al. The synovial sarcoma SYT-SSX2 oncogene remodels the cytoskeleton through activation of the ephrin pathway. Mol Biol Cell. 2007;18(10):4003-4012.

14. Charville GW, et al. EWSR1 fusion proteins mediate PAX7 expression in Ewing sarcoma. Mod Pathol. 2017;30(9):1312-1320.

15. Huang da W, Sherman BT, Lempicki RA. Systematic and integrative analysis of large gene lists using DAVID bioinformatics resources. Nat Protoc. 2009;4(1):44-57.

16. Parry D, et al. Dinaciclib (SCH 727965), a novel and potent cyclin-dependent kinase inhibitor. Mol Cancer Ther. 2010;9(8):2344-2353.

17. Desai BM, et al. The anti-melanoma activity of dinaciclib, a cyclin-dependent kinase inhibitor, is dependent on p53 signaling. PLOS ONE. 2013;8(3):e59588.

18. Fu W, et al. The cyclin-dependent kinase inhibitor SCH 727965 (dinacliclib) induces the apoptosis of osteosarcoma cells. Mol Cancer Ther. 2011;10(6):1018-1027.

19. Oyama R, et al. Generation of novel patientderived CIC-DUX4 sarcoma xenografts and cell lines. Sci Rep. 2017;7(1):4712.

20. Misra RN et al. N-(cycloalkylamino)acyl-2-aminothiazole Inhibitors of cyclin-dependent kinase 2. N-[5-[[[5-(1,1-dimethylethyl)-2-oxazolyl] methyl]thio]-2-thiazolyl]-4-piperidinecarboxamide (BMS-387032), a highly efficacious and selective antitumor agent. JMed Chem. 2004;47(7):1719-1728.

21. Caldon CE, Musgrove EA. Distinct and redundant functions of cyclin E1 and cyclin E2 in development and cancer. Cell Div. 2010;5:2.

22. Aytes A, et al. ETV4 promotes metastasis in response to activation of PI3-kinase and Ras signaling in a mouse model of advanced prostate cancer. Proc Natl Acad Sci U S A. 2013;110(37):E3506-E3515.

23. Watson $\mathrm{S}$, et al. Transcriptomic definition of molecular subgroups of small round cell sarcomas. J Pathol. 2018;245(1):29-40. 